
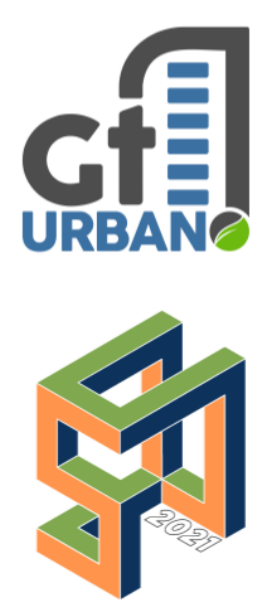

\section{SInGEURB}

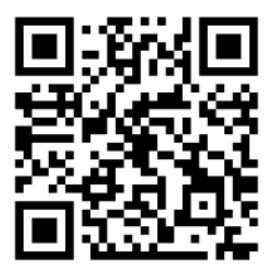

Como citar:

OTTONI, Christiano Costa Benedicto; OSCAR, Luiz Henrique Costa; QUALHARINI; Eduardo Linhares. Avaliação dos fatores de crescimento do setor de reabilitação urbana na cidade do Rio De Janeiro. In: III SIMPÓSIO

NACIONAL DE GESTÃO E ENGENHARIA URBANA: SINGEURB, 2021, Maceió. Anais... Porto Alegre: ANTAC, 2021. p. 487494.

Disponível em: https://eventos.antac. org.br/index.php/sin geurb/issue/view/14

\title{
Avaliação dos fatores de crescimento do setor de reabilitação urbana na cidade do Rio De Janeiro
}

\section{Evaluation of growth factores in the urban rehabilitation sector the city of Rio de Janeiro}

Christiano Costa Benedicto Ottoni, Universidade Federal do Rio de Janeiro, chrisottoni@poli.ufrj.br Luiz Henrique Costa Oscar, Universidade Federal do Rio de Janeiro, lhcosta@poli.ufrj.br

Eduardo Linhares Qualharini, Universidade Federal do Rio de Janeiro, qualharini@poli.ufrj.br

\section{RESUMO}

Neste artigo é apresentado um levantamento dos fatores que influenciam essa tendência de crescimento para se analisar o potencial das práticas de reabilitação na cidade do Rio de Janeiro. Neste levantamento buscaram-se os conceitos e premissas que caracterizam os termos que compõe o processo de reabilitação para em seguida relacionar e desenvolver os fatores apontados por autores de estudos relacionados ao tema, como as influências destes para a tendência de crescimento da área. A análise de como tais fatores se relacionam com a cidade do Rio de Janeiro é realizada a partir da correlação com as características econômicas, sociais, urbanísticas e políticas da cidade, indicando um cenário promissor para as práticas de reabilitação urbana nos próximos anos, aderente às especificidades locais e se apresentando como uma alternativa para redução dos impactos causados no setor da construção civil.

Palavras-chave: Reabilitação Urbana, Construção Civil, Sustentabilidade.

\begin{abstract}
This article aims to survey the factors that influence this growth trend to analyze the potential of rehabilitation practices in the city of Rio de Janeiro. In this survey, the concepts and premises that characterize the terms that make up the rehabilitation process were sought in the first instance, to then relate and develop the factors pointed out by authors of studies related to the theme, as influences for the growth trend of the area. The analysis of how such factors are related to the city of Rio de Janeiro is made from the correlation with the economic, social, urban and political characteristics of the city and indicate a promising scenario for urban rehabilitation practices in the coming years, apparently adherent local specificities, as an alternative to reduce the impacts caused in the civil construction sector.
\end{abstract}

Keywords: Urban Rehabilitation, Civil Construction, Sustainability. 


\section{INTRODUÇÃO}

A construção civil, que pode ser caracterizada como a atividade produtiva da construção que envolve a instalação, reparação, equipamentos e edificações, é um dos setores mais importantes para a economia do Brasil, tendo no período de 2011 a 2013 respondido por 4,9\% do PIB (OLIVEIRA, 2012). Dentro deste cenário, Qualharini (2017) indica que uma das áreas que apresenta maior tendência de crescimento no país é a de reabilitação urbana. De acordo com Croitor (2008), a reabilitação de edifícios não está limitada somente às edificações antigas e degradadas, mas também a outros grupos que potencialmente podem integrar esse mercado: edificações que se encontram inacabadas e abandonadas, na substituição de sistemas prediais ineficientes e na mudança de uso do imóvel.

Assim, este artigo traz os principais conceitos e premissas relacionados à reabilitação urbana, caracterizando os termos que compõe o seu processo e um levantamento de fatores e suas influências, que são apontados em estudos relacionados ao tema. A análise foi desenvolvida a partir da revisão de literatura quanto ao aumento desta tendência, propondo a discussão de sua aplicação em áreas metropolitanas a partir do contexto econômico, social, urbanístico e político da cidade.

\section{REABILITAÇÃO URBANA}

A reabilitação urbana pode ser definida como o conjunto de operações dirigidas à conservação e ao restauro das partes significativas de um patrimônio, incluindo a sua beneficiação, de forma a permitir o alcance de níveis de desempenho e exigências funcionais atualizadas, podendo ser aplicado tanto no contexto de intervenções urbanas quanto no contexto de um edifício (PAIVA; AGUIAR; PINHO, 2006).

Além de empregar o conhecimento da técnica construtiva, o conjunto de possibilidades para adoção de novos materiais de execução, o estudo da cultura construtiva local e a inserção na malha urbana existente, a reabilitação urbana conduz a valorização do parque edificado, contribuindo na requalificação dos espaços edificados, antes abandonados e degradados (QUALHARINI, 2017). Assim, indica a análise da opção mais adequada para a intervenção no bem edificado promovendo novos usos e consequente requalificação de áreas ora devolutas (MOTA, 2018). Dessa maneira, a figura 1 ilustra como a área da reabilitação, urbana e de edifícios, se contextualiza dentro da atividade produtiva da construção civil.

Figura 1 - Contexto da construção civil associado à reabilitação urbana.

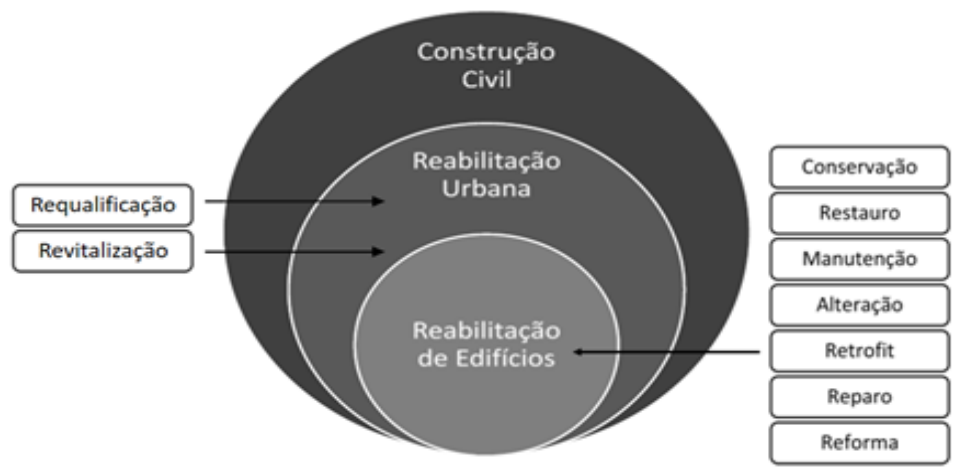

Fonte: Jesus (2008) 
Enquanto a requalificação e a revitalização são atividades relacionadas à Reabilitação de espaços urbanos, as intervenções relacionadas à reabilitação de edifícios, apesar de direcionadas a benfeitorias, também aumentam a percepção da população quanto à melhoria das condições da região. Isso acontece, pois, a reabilitação de edifícios promove oportunidades para valorização de seu entorno, à medida que possibilita por exemplo, o reforço da função social de determinada região, devido ao incremento de ações para a demanda da produção habitacional (JESUS, 2008). Segundo Jesus (2008), é necessário cumprir exigências como: Elaboração de projetos em edifícios reabilitados que contemple seu entorno; Observância de requisitos tecnológicos para intervenções; e, a indicação da logística de sua produção, que é diferente da dinâmica de construções novas.

Portanto, a definição de uma estratégia de reabilitação é um processo complexo que determina o grau de conhecimento e importância da área à intervir. Esse processo é comandado por fatores inerentes à construção em si (estado em que se encontra maior ou menor facilidade para adaptação às novas condições, vida útil remanescente, encargos futuros de manutenção), mas também por fatores externos (plano de negócios atual do proprietário e sua evolução futura, rentabilidade permitida pela exploração da construção, valor fundiário atual, valorização futura), ou seja, a estratégia a adotar é comandada por aspectos de índole política, social, cultural, legal e econômica (CÓIAS, 2006).

\section{FATORES QUE INFLUENCIAM O CRESCIMENTO DO SETOR}

Em vista desse complexo processo para sua implementação, que exige a consideração de aspectos diversos para a tomada de decisão, são levantados os principais fatores e suas influências, apontados em estudos relacionados ao tema, que justificam a tendência de crescimento do setor da reabilitação dentro da atividade produtiva da construção civil.

\subsection{Baixo estoque de terrenos disponíveis nos centros urbanos.}

Nos grandes centros urbanos onde há baixo estoque de terrenos disponíveis para uma nova construção, a reabilitação predial se apresenta como uma alternativa que possibilita impactos econômicos, sociais e ambientais. Outro aspecto versa sobre a necessidade de habitação de classes trabalhadoras, que foi parte de debates arquitetônicos e urbanísticos desde o século XIX. Essa tipologia definiu as bases sobre as quais se estruturaram as cidades modernas, com seu dogmatismo na separação das funções da cidade: habitar, circular, trabalhar e divertir-se (OTERO, 2010).

\subsection{Idades elevadas e obsolescência das edificações.}

A vida útil de projeto (VUP), segundo a ABNT (2011), é o período estimado de tempo em que um sistema é projetado para atender aos requisitos de desempenho, desde que o programa de manutenção seja cumprido. Qualharini (2017) reforça que a vida útil das edificações é de aproximadamente 80 anos, sendo importante considerar que existe a tendência de aumento no estoque de imóveis com idades passíveis de intervenção. Como exemplo, a partir de estudo que vem sendo atualizado desde o ano 2000 pelo Instituto Pereira Passos, apurou se em 2019 que 39\% das edificações da cidade do Rio de Janeiro possuíam de 0 a 20 anos, resultando em um estoque de 61\% de imóveis com idades já passíveis de intervenção (IPP, 2019), indicado na figura 2. 
Figura 2 - Imóveis prediais por faixa de idade na cidade do Rio de Janeiro

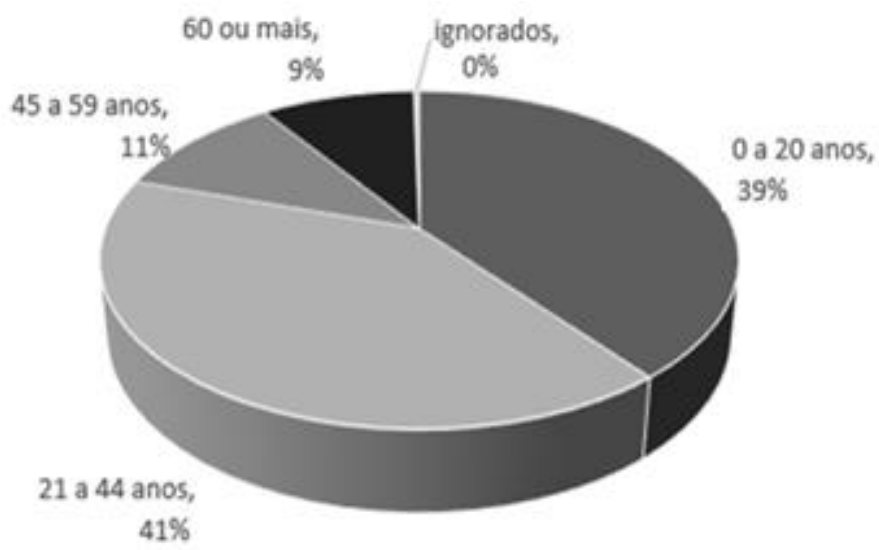

Fonte: IPP (2019)

\subsection{A contribuição da Sustentabilidade na Reabilitação Urbana}

Vale (2006) indica que a reabilitação das edificações pode incorporar parâmetros para a sustentabilidade, evitando o descarte de um patrimônio, sendo este reaproveitado, ampliado melhorado e contribui por deixar de criar toneladas de resíduos, tanto no descarte do antigo, quanto na construção de uma nova edificação, afirmou Kruger (2016).

Cianciardi (2004), afirma que parâmetros sustentáveis para a construção não almejam apenas o resguardo de direitos do ecossistema, mas buscam um edifício saudável, que propicie proteção, conforto e salubridade ao ser humano.

As exigências do mercado sustentável incluem, segundo Correia (2011), a capacidade de reconhecer e desafiar as barreiras no conhecimento de novos produtos e ofertar tecnologias alternativas com o uso de processos que visem à eficiência orgânica dos empreendimentos. Estas exigências indicam que práticas sustentáveis são preponderantes para incremento de ações de reabilitação urbana.

\subsection{Custo das obras de Reabilitação (Prédio Novo x Reabilitação)}

Baseando-se em uma análise econômica, Cantanhede (2003) afirmou que o custo unitário básico é um índice, criado em função da Lei nº 4.591 (BRASIL, 1964), com a finalidade de auxiliar na avaliação dos custos de incorporação antes do lançamento de empreendimentos, já nas fases iniciais de concepção onde existem poucas informações sobre o empreendimento. Porém, não existe metodologia para estimar o custo das diversas facetas das obras de reabilitação.

Torres (2015) diz que em Portugal, embora a indústria da construção esteja na atualidade redirecionada para projetos de reabilitação, também não existem "bases de dados" disponíveis e preços comparáveis de região para região. Para suprir a dificuldade, o autor realizou cinco estudos de caso na cidade da Covilhã para verificar o custo global de uma intervenção, comparando sempre com uma nova construção do mesmo partido. 
A determinação dos custos de reabilitação por metro quadrado para cada caso de estudo abordado por Torres (2015) permitiu encontrar um valor médio global e verificar que a intervenção de reabilitação teria uma economia de $30 \%$ em comparação ao custo de uma nova.

\subsection{Déficit Habitacional Brasileiro}

O processo de desenvolvimento de cidades brasileiras apresenta realidades urbanas distintas, uma caracterizada por alta renda, que apresentam qualidade urbanística e ambiental, e outra por extensas áreas habitadas pelas camadas populares, que são carentes de equipamentos e serviços públicos, infraestrutura urbana, postos de trabalho e consumo, áreas de lazer, entre outros (OTERO, 2010).

A Fundação João Pinheiro (2018), apontou que o déficit habitacional estimado correspondia a 6,355 milhões de domicílios, onde 5,572 milhões ou 87,7\% estão em áreas urbanas. De acordo com o IBGE (2015) na Pesquisa Nacional por Amostra de Domicílios, 7,906 milhões de imóveis vagos, 80,3\% localizados em áreas urbanas. Desse total, 6,893 milhões estão em condições de serem ocupados e 1,012 milhão estão em construção ou reforma. Esses dados apresentam uma dimensão contrária às premissas da Constituição Federal de 1988 (CF88). No entanto, somente em 2001 com a aprovação do Estatuto da Cidade, Lei 10.257/01, foram regulamentados instrumentos para o uso da propriedade urbana em prol do bem coletivo.

Segundo Rodrigues (2004), o Estatuto induziu o reconhecimento da cidade como produção coletiva, reafirmou a propriedade privada/individual, impôs limites à especulação e criou instrumentos jurídicos e participativos que permitem ao poder público tomar providências, para que as propriedades cumpram sua função social em prol do bem coletivo, da segurança e do bem-estar dos cidadãos. Dentro destes aspectos, o reconhecimento do déficit habitacional apresenta demandas para novas construções que poderiam ser supridas com a adoção de práticas de reabilitação no estoque de domicílios vagos impactando na renovação urbana.

\section{ANÁLISE}

As grandes cidades brasileiras, em especial àquelas onde o processo de urbanização aconteceu no início do século XX, se apresentam como promissores exemplos urbanos onde as práticas de reabilitação urbana devem ser estimuladas ao longo dos próximos anos. Seja pela indisponibilidade de terrenos nas áreas mais valorizadas da cidade, seja pela idade avançada do parque edificado onde $61 \%$ dos imóveis estão passíveis de intervenções, como é o caso da cidade do Rio de Janeiro, ou ainda, pelos aspectos relacionados à sustentabilidade que são cada vez mais exigidos pela sociedade, assim, existe uma tendência de crescimento no país da prática de intervenções relacionadas à reabilitação urbana.

Também, é possível apontar a reabilitação urbana como uma alternativa ao setor da construção civil, viável social e economicamente, que pode promover a preservação e ressignificação do patrimônio urbano cultural. Com esta premissa, há a necessidade de documentar a escolha do desempenho ideal do envelope urbano, estabelecendo planos diretores que possam premiar a dinâmica de crescimento das microrregiões, a partir da proposta de políticas de renovação e reabilitação urbana de médio e longo prazos, de forma a reconhecer a vocação regional urbana em função da cidade contemporânea.

Ainda que de forma isolada já existem registros de intervenções que, mesmo estando associadas à mitigação do déficit habitacional, representaram iniciativas relevantes de intervenções que trouxeram impactos positivos para suas respectivas regiões. Tais intervenções lidaram com as dificuldades de reabilitação 
quanto à idade do imóvel, a fase elevada de seu ciclo de vida e questões orçamentárias complexas, porém com a elaboração de projetos específicos foi possível superar as barreiras quanto à ausência de estoque de terrenos disponíveis e adotar alternativas sustentáveis de intervenção. Ao observar os resultados comerciais positivos obtidos por estas intervenções isoladas, pode-se afirmar que já está disponível conhecimento técnico para realizar intervenções relacionadas à reabilitação urbana, contribuindo para que cada edifício reabilitado alcance indicadores de sustentabilidade mais eficientes do que os não reabilitados, o que permite, por exemplo, a utilização de recursos como a água de forma permanente, redução no consumo de energia e a integração destas ações com políticas de consumo consciente pela sociedade.

\section{CONCLUSÃO}

Nas grandes cidades brasileiras existem iniciativas isoladas que premiam ações de patrimônio, indicando um cenário que pode ser explorado como alternativa de negócios na construção civil. Para tal, os fatores citados nesse artigo são exemplos que podem ser beneficiados com a reabilitação urbana, podendo contribuir para trazer intervenções que favoreçam a valorização da cidade. Assim, a divulgação de estudos e resultados neste âmbito provoca o interesse da sociedade, ao passo que criam oportunidades para continuidade ou surgimento de novas intervenções. Porém, se faz necessário cauleta na adoção destas práticas, pois há desvantagens que devem ser mapeadas para verificação da viabilidade destas intervenções no bem edificado. Estas são associadas aos riscos das soluções encontradas, ao tempo que durarão, aos materiais que serão poupados, a quantidade de energia que será consumida, se de fato apresentarão resultados monetários e se contribuirão para o novo uso e de operação do patrimônio.

É possível encontrar soluções que já são estão consolidadas nas cidades, devendo ser aprimoradas, de maneira a permitir a adequação às necessidades urbanas e à continuidade na abertura de caminhos eficientes para o segmento. Dentro destes aspectos, as ações se aderem à relevância quanto ao estudo da reabilitação urbana, confirmando os fatores que influenciam o crescimento do setor e sua aplicabilidade nas grandes cidades brasileiras. Por fim, as ações de reabilitação podem ser um indício para um mercado promissor e crescente, que pressionado pelo desejo de renovação urbana, promova a valorização e preservação do patrimônio edificado. Adicionalmente, a adoção das práticas de readequação de edificações se apresentam como alternativa para redução dos impactos causados no setor da construção civil de modo a consolidar práticas sustentáveis, promover a melhoria das condições do entorno edificado e incrementar a qualidade de vida na região da intervenção.

\section{REFERÊNCIAS}

ASSOCIAÇÃO BRASILEIRA DE NORMAS TÉCNICAS. ABNT NBR 14037: Manual de operação, uso e manutenção das edificações. Rio de Janeiro: ABNT, 2011.

BRASIL. Constituição da República Federativa do Brasil de 1988. Brasília, DF: Presidência da República. Disponível em: https://www2.senado.leg.br/bdsf/bitstream/handle/id/518231/CF88_Livro_EC91_2016.pdf . Acesso em: 25 de jun. 2020.

CANTANHEDE, D. A. G. Custos unitários básicos (CUB): verificação e validação do modelo de cálculo. 2003. Dissertação (Pós-graduação em Engenharia Civil) - Escola de Engenharia, UFRGS, 2003. Disponível em: https://www.lume.ufrgs.br/bitstream/handle/10183/3458/000388637.pdf?sequence=1 . Acesso em 11 jun. 2020. 
CIANCIARDI, G.; MONTEIRO, R. Z.; BRUNA, G. C. Parâmetros de sustentabilidade ecológicos na recuperação, manutenção e restauração de edifícios. IV Seminário Internacional da Lares Latim America Real Estate Society, São Paulo, Brasil, 2004.

CORREIA. M. C, QUALHARINI, E. L.;. A Gestão do Projeto na Reabilitação e Manutenção Sustentável de Edifícios. In: $6^{0}$ Congresso Luso-Moçambicano de Engenharia, 2011, Maputo. $6^{0}$ Congresso LusoMoçambicano de Engenharia, 2011.

CÓIAS, V.; SOARES, I. A revisão dos projectos como forma de reduzir os custos da construção e os encargos da manutenção de edifícios. 3o ENCORE, 2003, 1347 a 1354, Lisboa.

CROITOR, E. P. N. A gestão de projetos aplicada à reabilitação de edifícios: estudo da interface projeto e obra. 2002. 176 f. Dissertação (Mestrado em Engenharia Civil) - Escola Politécnica, Universidade de São Paulo, São Paulo, 2008.

FJP, Centro de Estatística e Informações. Déficit habitacional no Brasil 2015. Belo Horizonte: Fundação João Pinheiro, 2018.

IBGE. Pesquisa nacional por amostra de domicílios: síntese de indicadores 2015. Rio de Janeiro: IBGE, 2016. Disponível em: https://servicodados.ibge.gov.br/Download/Download.ashx?http=1\&u=biblioteca.ibge.gov.br/visualizacao /livros/liv98887.pdf . Acesso em: 17 de jul. 2020.

INSTITUTO PEREIRA PASSOS (IPP). Armazém de Dados do IPP - Tabela 7.1.6 - Imóveis prediais e área construída, por faixa de idade, segundo suas tipologias nas Áreas de Planejamento e Regiões Administrativas. Rio de Janeiro, Brasil, 2000. Atualizada em 2019.

JESUS, C. R. M. Análise de Custos para Reabilitação de Edifícios para Habitação. 2008. Dissertação (Mestrado) - Escola Politécnica da Universidade de São Paulo, Universidade de São Paulo, São Paulo, 2008.

KRUGER, A.; SEVILLE, C.. Construção verde: princípios e práticas em construção residencial. Adaptação de S. H. Obata. Cengage Learning, São Paulo, 2016.

MOTA, C. A. Diretrizes construtivas na reabilitação sustentável de edifícios. 2018. Dissertação (mestrado) Escola Politécnica , Universidade Federal do Rio de Janeiro, Rio de Janeiro, 2018

OTERO, E. V.; MARTINS, M. L. R.. A reabilitação de conjuntos habitacionais na cidade de São Paulo. In: III Congresso Internacional na Recuperação, Manutenção e Restauração de Edifícios. Rio de Janeiro, Brasil, 2010.

PAIVA, J. V.; AGUIAR, J.; PINHO, A. Guia técnico de reabilitação habitacional. 1. ed. LISBOA: LNEC, 2006, 2 vol, $467 \mathrm{p}$.

QUALHARINI, E. L.. Reabilitação predial como alternativa para a sustentabilidade nas construções brasileiras. In: International Congress of Engineering, 2017, Covilha; Anais [...]. Covilha: Umiversity of Beira Interior, 2017. 
A Evolução da Reabilitação Predial no século XXI. In: 4ํ PATORREB 2012 - 4ํㅡㄹ Congresso sobre patologia e reabilitação de edifícios, 2012, Santiago de Compostela. Colexio Oficial de Arquitectos da Galicia, 2012.

RODRIGUES, A. M.. Estatuto da cidade: função social da cidade e a propriedade. Alguns aspectos sobre população urbana e espaço. Cadernos Metropole, n. 12, p. 9-25, 2004.

TORRES, A. Empreendimentos de construção e reabilitação: custos associados. Dissertação - Universidade da Beira Interior, Covilhã, Portugal, 2015.

VALE, M.S. Diretrizes para racionalização e atualização das edificações: Segundo o conceito da qualidade e sob a ótica do Retrofit. 2006. Dissertação (Mestrado) - Programa de Pós-graduação em Arquitetura, Faculdade de Arquitetura e Urbanismo, Universidade Federal do Rio de Janeiro, Rio de Janeiro, 2006. 